\title{
EPIDEMIOLOGIA DO CÂNCER DE COLO DE ÚTERO: UMA REALIDADE DA SAÚDE PÚBLICA DO PARANÁ
}

\section{EPIDEMIOLOGY OF CERVICAL CANCER: A PUBLIC HEALTH ANALYSIS IN THE STATE OF PARANÁ}

\author{
Francisco José Koller'1, Michelle de Lima ${ }^{1}$, Gisele Cristina de Campos Cruz ${ }^{2}$, Paulo \\ Henrique Peixoto ${ }^{1}$, Nádia Vercka Novak ${ }^{3}$ \\ ${ }^{1}$ Faculdades Integradas Santa Cruz. Curitiba, Paraná, Brasil, ${ }^{2}$ Fundação Estatal de \\ Atenção Especializada de Saúde, ${ }^{3}$ Hospital Erasto Gaertner, Curitiba, Paraná, Brasil \\ *Autor correspondente: Francisco José Koller. Rua Dilermando Pereira de Almeida, 71. \\ CEP: 81870-070 - Curitiba - Paraná, Tel.: (41) 99980-8863. E-mail: enfkoller@yahoo.com. \\ br/ enfkoller@gmail.com
}

\section{RESUMO}

O objetivo desta pesquisa é traçar o perfil epidemiológico das mulheres acometidas pelo câncer de colo de útero. Trata-se de uma abordagem epidemiológica, descritiva e retrospectiva, com base no banco de dados do Instituto Nacional de Câncer José Alencar Gomes da Silva (INCA), no período de janeiro de 2005 a dezembro de 2014, através da análise das variáveis disponíveis no sistema Registro Hospitalar de Câncer (RHC). Os resultados mostraram que o câncer de colo de útero ocorreu em mulheres principalmente na faixa etária entre 30 e 39 anos, da cor branca, solteiras, com ensino fundamental incompleto, com histórico de alcoolismo (46\%) e tabagismo (59\%). 41\% delas eram trabalhadoras dos serviços de vendas do comércio em lojas e mercados, sendo que $66 \%$ tiveram como exame diagnóstico a anatomia patológica. Quanto ao tipo histológico predominaram os carcinomas (38\%), nos quais foram utilizados como primeiro tratamento a cirurgia (49\%). Verificou-se que o rastreamento e a detecção precoce do câncer de colo de útero, assim como as ações preventivas voltadas para o incentivo à realização do exame citopatológico, são eficazes para minimizar o número de novos casos e de diagnósticos tardios.

Palavras-chaves: Epidemiologia; Câncer de Colo Uterino; Enfermagem; Banco de Dados.

\begin{abstract}
The present study aims to determine the epidemiological profile of women affected by cervical cancer. This is an epidemiological, descriptive and retrospective study based on data obtained at the José Alencar Gomes da Silva National Cancer Institute (INCA) database, from January 2005 to December 2014, through analysis of the variables available at the Hospital Cancer Registry Information System (RHC). According to the results, cervical cancer occurred in white, single women aged 30-39 years, with incomplete primary education, history of alcohol use (46\%) and smoking (59\%). Also, $41 \%$ of them worked in shops and grocery stores, and the diagnostic test of $66 \%$ was anatomic pathology. As for the histologic type, carcinoma was prevalent (38\%), and surgery was the preferred procedure to treat the disease (49\%). Screening and early detection of cervical cancer, as well as preventive actions aimed to encourage women to perform the cytopathological examination effectively reduce the number of new cases and late diagnoses.
\end{abstract}

Keywords: Epidemiology; Cervical Cancer; Nursing; Database. 


\section{INTRODUÇÃO}

O número de novos casos de câncer no Brasil vem aumentando no decorrer das últimas décadas devido às alterações da saúde da população. Tais transformações provocaram aumento da expectativa de vida, urbanização acelerada, alimentação inadequada, hábitos de vida inapropriados e exposição dos indivíduos a fatores ambientais negativos nos âmbitos físico, químico e biológico, prejudicando a saúde ${ }^{1}$.

O câncer de colo de útero representa um grave problema de saúde pública, em especial nos países em desenvolvimento, que apresentam cerca de $80 \%$ dos casos de mortes em virtude desta patologia ${ }^{2}$.

No Brasil observou-se um crescimento no número de mulheres acometidas por esse câncer, com um aumento no número de casos e ocorrência em idades cada vez mais precoces, além da elevação da taxa de mortalidade ${ }^{3}$.

No ano de 2016, o Instituto Nacional de Câncer José Alencar Gomes da Silva (INCA) apresentou uma estimativa de 16.340 novos casos de câncer de colo uterino, aproximadamente 15,85 casos para cada 100 mil mulheres, sendo que a região Sul ocupa a terceira posição, com 15,17/100 mil'1 .

Segundo a Organização Mundial de Saúde, os métodos utilizados para a detecção precoce do câncer de colo uterino incluem o diagnóstico precoce, baseado nos sinais e sintomas, e o rastreamento através do exame citopatológico, com o intuito de identificar lesões sugestivas de câncer e tratá-las antes que a doença se desenvolva ${ }^{4}$.

O câncer de colo uterino apresentou um aumento da morbidade e mortalidade, sendo este um problema que tem levantado uma importante questão em relação à saúde das mulheres, pois a prevenção e a detecção precoce são fatores essenciais para a diminuição dos índices de mortalidade 5 .

A baixa procura para a realização do exame citopatológico se deve a fatores socioeconômicos e culturais, como baixa escolaridade, baixa renda familiar e falta de informação. Outros fatores citados pelas mulheres foram a vergonha, o medo do procedimento, o resultado do exame, o preconceito, a exposição e a falta de orientação, fazendo com que elas não procurem o serviço por não terem ciência da importância deste exame ${ }^{6}$.

Por isso é indispensável a participação dos profissionais de saúde para investir na educação nessa área, ficando clara a necessidade da equipe em tentar mudar este tabu que ainda existe em relação à realização do exame citopatológico ${ }^{6}$.

Considera-se de grande importância e também um grande desafio desenvolver estratégias e técnicas que possam permitir a detecção e o diagnóstico precoce da neoplasia do colo uterino, para, desta forma, diminuir a taxa de mortalidade. Se todas as mulheres tivessem acesso ao rastreamento, diagnóstico, tratamento e monitoramento adequado, isso seria essencial para a diminuição da taxa de mortalidade e morbidade ${ }^{7}$.

Diante do conteúdo exposto neste estudo, surge a necessidade de analisar o perfil das mulheres acometidas pelo câncer de colo de útero no Paraná.

\section{METODOLOGIA}

Trata-se de uma abordagem epidemiológica descritiva e retrospectiva, utilizando os dados secundários do sistema informatizado integrador Registro Hospitalar de Câncer (RHC) do estado do Paraná, disponibilizado pelo Instituto Nacional de Câncer José Alencar Gomes da Silva (INCA), no período de janeiro de 2005 a dezembro de 2014.

A coleta de dados ocorreu através da utilização do ICD-10 representado pelo C53: Colo de útero, no campo "localização primária", e pelo ano em que foi registrado o caso.

Para descrever a tendência linear por período, foram calculados a variação percentual anual (VPA) e seus respectivos intervalos de confiança de $95 \%$ (IC95\%). O estudo foi aprovado pelo Comitê de Ética em Pesquisa do Instituto de Otorrinolaringologia do Paraná (CAAE: 61755616.8.0000.5529/Parecer: 1.841.132).

\section{RESULTADOS}

No estado do Paraná, no período de 2005 a 2014, foram registrados 12.574 casos de câncer de colo de útero. A idade variou de 22 a 95 anos, com predomínio da faixa etária de 30 a 39 anos (26,0\%), seguida da de 40 a 49 anos $(20,8 \%)$, da raça branca $(85,4 \%)$, mulheres solteiras $(39,7 \%)$, com ensino fundamental incompleto $(34,9 \%)$ e com histórico de alcoolismo $(46,8 \%)$ e tabagismo $(59,7 \%)$.

Em relação às características do câncer de colo de útero (Tabela 1), verifica-se que a agressividade da patologia é alarmante, pois o rastreamento precoce possibilita a identificação de alterações celulares e tratá-las antes que a doença se desenvolva. 
Tabela 1 - Característica do câncer de colo de útero das mulheres paranaenses, Paraná, Brasil, 2005-2014

\begin{tabular}{c|c|c|c}
\hline \multirow{2}{*}{ CARACTERÍSTICA } & n. & $\%$ \\
\hline Localização do Tumor & Neoplasia maligna do colo do útero, não especificado & 9890 & 78,7 \\
\hline & Neoplasia maligna do exocérvix & 1496 & 11,9 \\
\hline & Neoplasia maligna do endocérvix & 953 & 7,6 \\
\hline & Neoplasia maligna do colo do útero com lesão invasiva & 235 & 1,9 \\
\hline Tipo Histológico & Carcinomas gerais & 4814 & 38,3 \\
\hline & Outras histologias & 3514 & 27,9 \\
\hline & Carcinoma não especificado & 2780 & 22,1 \\
\hline & Adenocarcinoma não especificado & 893 & 7,1 \\
\hline & Carcinoma escamoso ou epidermoide & 510 & 4,1 \\
\hline & Outros adenocarcinomas & 27 & 0,2 \\
\hline & Adenocarcinoma de células claras & 23 & 0,2 \\
\hline & Adenocarcinoma endometrioide & 4 & 0,0 \\
\hline & Adenocarcinoma mucinoso & 2 & 0,0 \\
\hline & Sem informação & 6 & 0,0 \\
\hline & Não se aplica** & 7515 & 59,8 \\
\hline & Estádio I & 1420 & 11,3 \\
\hline & Estádio II & 1382 & 11,0 \\
\hline & Estádio 0 & 1011 & 8,0 \\
\hline & Estádio IV & 270 & 2,1 \\
\hline & Estádio III & 55 & 0,4 \\
\hline & Sem informação* & 922 & 7,3 \\
\hline & Total & 12574 & 100 \\
\hline & & &
\end{tabular}

Fonte - RHC INCA (2016)

*Item não preenchido devido à falta de informação no prontuário médico.

**Quando o tumor não for estadiável.

\section{DISCUSSÃO}

Os registros de câncer de colo de útero são considerados uma fonte informativa da condição de saúde da mulher paranaense, pois os dados são gerados pelas instituições hospitalares que fazem a propedêutica das pacientes oncológicas, tendo por obrigatoriedade a transmissão dos casos para o Ministério da Saúde através do sistema Siscolo e RHC ${ }^{8}$.

As projeções apontam que o câncer de colo de útero pode acometer mulheres principalmente a partir dos 30 anos, aumentando sua gravidade até atingir mulheres com mais de 50 anos, tendo sido responsável pelo óbito de 265 mil mulheres em 2012, representando $87 \%$ dos casos em países em desenvolvimento ${ }^{1-9}$.

A condição de saúde pode ser responsável pela falta de entendimento e conhecimento sobre a importância do exame citopatológico, indicando que tal condição pode interferir de forma negativa para o diagnóstico, tratamento precoce e prevenção do câncer de colo uterino ${ }^{3-10}$.

A associação do risco de contrair o câncer de colo de útero com o nível de escolaridade sugere que quanto menor for o grau de escolaridade da população, maior será o risco de se desenvolver a neoplasia do colo de útero, devido às dificuldades enfrentadas, como a falta de informação e conhecimento sobre o rastreamento, o tratamento precoce e a dificuldade de locomoção até os serviços de saúde, o que demonstra a necessidade de se investir em ações de promoção e prevenção ${ }^{11}$.

Em um estudo realizado em Roraima, do qual participaram 603 mulheres, $46 \%$ possuíam o ensino médio completo, $1,6 \%$ eram analfabetas e $28,6 \%$ tinham nível superior completo, constatando-se que o nível de instrução das participantes não foi alterado em relação à realização do exame citopatológico ${ }^{12}$. 
Corroborando os dados sobre qualidade de vida, observou-se que os piores fatores, como o tabagismo, a obesidade e o consumo exagerado de álcool, interferem na saúde e nas atividades cotidianas, dificultando a prática de atividade física, uma alimentação saudável e a saúde bucal, podendo acarretar outras patologias associadas ao uso destas substâncias, como problemas circulatórios, cardíacos, neoplasia de pulmão, transtornos mentais ${ }^{13}$.

Verificou-se que as mulheres fumantes têm maior possibilidade de apresentar alterações celulares, pois os epitélios cervicais possuem menor número de células imunitárias do que em não fumantes, o que facilita a proliferação de lesões causadas por vírus ${ }^{6}$.

A infecção pelo papilomavírus humano é uma condição necessária em quase todos os casos de câncer de colo uterino. É uma doença sexualmente transmissível, e todas as mulheres sexualmente ativas correm o risco de serem infectadas. Existem atualmente três vacinas contra o papilomavírus humano, e apesar da eficácia ser bem comprovada em mulheres com idade até 25 anos, não está bem estabelecida em idades mais avançadas ${ }^{14}$.

Alguns fatores influenciam as mulheres para a não realização periódica deste exame, como a dificuldade em ter acesso aos serviços de saúde, falta de informação, envolvimento do profissional, tempo de espera para realizar o agendamento e ainda fatores relacionados aos sentimentos, tais como medo, vergonha, ansiedade e timidez ${ }^{15}$.

Em uma pesquisa realizada em Cajazeiras sobre a percepção das mulheres em relação ao câncer de colo de útero, constatou-se que $52 \%$ entendiam a neoplasia como um tipo de patologia grave que causa uma ferida no útero, $8 \%$ relataram ser uma patologia sem cura, $20 \%$ achavam que a patologia tinha cura, mas se não tratada levava à morte, e $12 \%$ não sabiam sobre esta patologia ${ }^{16}$.

Observou-se que havia uma falta de conhecimento destas mulheres em relação à patologia, possivelmente devido à ausência de orientação e conhecimento repassados pelos profissionais de saúde ${ }^{16}$.

Esses dados nos levam a considerar uma possível falha no diagnóstico, na coleta do exame preventivo ou na dificuldade de interpretação dos resultados, já que $35 \%$ das mulheres referiram fazer o exame periodicamente com o intervalo que é recomendado ${ }^{9-17}$. Apenas 30\% destas mulheres relataram que fizeram o exame citopatológico pelo menos três vezes na vida, o que pode resultar no diagnóstico já em fase avançada, que ocorre em cerca de $70 \%$ dos $\operatorname{casos}^{17}$.

\section{CONCLUSÃO}

O aumento do número de novos casos da neoplasia maligna de colo uterino pode ser evitado através do rastreamento, que é realizado pela coleta do exame citopatológico, e da detecção precoce, constituindo as formas mais simples e utilizadas para se diagnosticar a doença. Assim sendo, quanto mais cedo essas alterações celulares forem identificadas, melhor poderão ser tratadas, evitando que a doença se desenvolva e aumentando as possibilidades de cura.

É importante salientar que as ações de promoção e prevenção voltadas para a saúde das mulheres, como o incentivo e a busca ativa para realização periódica do exame citopatológico, são extremamente relevantes. Os cuidados, a detecção precoce e a aceitação do tratamento contribuem para a cura e o aumento da taxa de sobrevida, consequentemente diminuindo os casos de óbitos ocasionados por esta patologia.

A atuação do enfermeiro, como profissional do cuidado, é fundamental para dar suporte no diagnóstico, tratamento, orientação e apoio emocional a estas pacientes, auxiliando no que for possível para minimizar os danos, tanto físicos como psicológicos, que esta doença pode ocasionar na vida destas mulheres.

\section{REFERÊNCIAS BIBLIOGRÁFICAS}

1 BRASIL. Instituto Nacional do Câncer José Alencar Gomes da Silva- INCA. Estimativa | 2016 Incidência de Câncer no Brasil. Rio de Janeiro, 2016. [citado em 2016 dez 10]. Disponível em: www.inca.gov.br/wcm/dncc/2015/ estimativa-2016.asp

2 ALBUQUERQUE, K.M et al. Cobertura do teste de Papanicolau e fatores associados à não-realização: um olhar sobre o Programa de Prevenção do Câncer do Colo do Útero em Pernambuco, Brasil. Caderno Saúde Pública, Rio de Janeiro.2009; 25(2):301-309. [citado em 2016 dez 17]. Disponível em: www.scielo.br/scielo.php?script $=$ sci arttext\&pid $=$ S0102-311X2009001400012

3 BIM, C.R et al. Diagnóstico precoce do câncer de mama e colo uterino em mulheres do município de Guarapuava, PR.Revista Escola Enfermagem USP. 2010; 44(4):940-6. [citado em 2016 dez 17]. Disponível em: $\quad$ www.scielo.br/scielo.php?script=sci_arttext\&pid $=$ S0080-62342010000400012. 
4 BRASIL. Instituto Nacional do Câncer José Alencar Gomes da Silva- INCA. ABC do câncer: abordagens básicas para controle de câncer. Rio de Janeiro, 2011. [citado em 2016 dez 05]. Disponível em: bvsms.saude.gov.br/bvs/ publicacoes/inca/abc_do_cancer_2ed.pdf

5 PELlOSO, A.S.; CARVALHO, M.D.B.; HIGARASHI, I.H. Conhecimento das mulheres sobre o câncer cérvicouterino. Acta Scientiarum. Health Sciences, Maringá, 2004; 26(2):319-324. [citado em 2016 dez 10]. Disponível em: https://www.nescon.medicina.ufmg.br/biblioteca/ imagem/0261.pdf

6 MEDEIROS T. et al. Conhecimento e percepção de mulheres quanto ao exame preventivo para o câncer de colo do útero. Revista Brasileira de Educação e Saúde, Pombal, 2015; 5(4): 09-16. [citado em 2016 dez 10]. Disponível em: $\quad$ www.scielo.br/scielo.php?script=sci_arttext\&pid $=$ S1413-81232007000300024

7 GIRIANELLI, V.R.; GAMARRAL, C.J.; SILVA, G.A. Os grandes contrastes na mortalidade por câncer do colo uterino e de mama no Brasil. Revista Saúde Pública, 2014;48(3):459467. [citado em $2016 \mathrm{dez}$ 17]. Disponível em: www.scielo. br/pdf/rsp/v48n3/pt_0034-8910-rsp-48-3-0459.pdf

8 BRASILInstituto Nacional do Câncer José Alencar Gomes da Silva- INCA. Registros hospitalares de câncer. Rotinas e procedimentos, Rio de Janeiro, 2012. [citado em $2016 \mathrm{dez}$ 17]. Disponível em: www2.inca.gov.br/.../inca_publica edicao_atualizada_manual_registros_hospitalares

9 DUSKA, L.R. Cervical Cancer: The Next Generation of Prevention, Detection, and Treatment. Clinical Therapeutics. 2016; 38(3): 446-448. [citado em 2016 dez 10]. Disponível em: http://dx.doi.org/10.1016/j.clinthera.2016.02.013

10 PINHO, F.J.R.; CHEIN, M.B.; THULER, L.C. Patterns of cervical cytological abnormalities according to the Human Development Index in the northeast region of Brazil. BMC Womens Health. 2016; 12;16:54. [citado em 2016 dez 10]. Disponível em: http:// 10.1186/s12905-016-0334-2.

11 DALLABRIDA, F.A et al.. Qualidade de vida de mulheres tratadas por câncer do colo de útero. Revista Rene, 2014;15(1):116-22. [citado em 2016 dez 17] Disponível em: www.revistarene.ufc.br/revista/index.php/revista/article/ viewFile/1487/pdf

12 NAVARRO C et al. Cobertura do rastreamento do câncer de colo de útero em região de alta incidência. Revista Saúde Pública, 2015;.49(17). [citado em 2016 dez 07]. Disponível em: www.scielosp.org/pdf/rsp/v49/pt_00348910-rsp-S0034-89102015049005554.pdf

13 OLIVEIRA-CAMPOS, $\mathrm{M}$ et al, Impacto dos fatores de risco para doenças crônicas não transmissíveis na qualidade de vida. Ciência \& Saúde Coletiva, 2013; 18(3):873-882. [citado em 2016 dez 10]. Disponível em: $\quad$ www.scielo.br/scielo.php?script=sci_arttext\&pid $=$ S1413-81232013000300033

14 RIBEIRO, J.P.; BORGES, I. Efficacy of the Vaccines Against Human Papillomavirus in Women Older than 24 Years in the Cervix Cancer Prevention. Acta Medica Portuguesa. 2016; 29(6): 401-08. [citado em 2016 dez 17]. Disponível em: http://actamedicaportuguesa.com/revista/ index.php/amp/article/view/7095/4718

15 SANTOS, U.M.; SOUZA, S.E.B. Papanicolau: diagnóstico precoce ou prevenção do câncer cervical uterino. Revista Baiana de Saúde Pública, 2013; 37(4):941951. [citado em 2016 dez 10]. Disponível em: rbsp.sesab. ba.gov.br/index.php/rbsp/article/view/420/0

16 SANTOS, M.S.; MACÊDO, A.P.N.; LEITE, M.A.G. Percepção de Usuárias de uma Unidade de Saúde da Família Acerca da Prevenção do Câncer do Colo do Útero. Revista APS, Juiz de Fora, 2010; 13(3):310-319. [citado em 2016 dez 10]. Disponível em: https://aps.ufjf.emnuvens.com.br/ aps/article/view/672

17 SOARES, M.C et al. Câncer De Colo Uterino: Caracterização das mulheres em um Município do Sul do Brasil. Escola Anna Nery Revista Enfermagem, 2010; 14(1):90-96. [citado em 2016 dez 17]. Disponível em: $\quad$ www.scielo.br/scielo.php?script=sci_arttext\&pid $=\mathrm{S} 1414-81452010000100014$ 